\title{
Tanshinone IIA regulates microRNA-125b/foxp3/caspase-1 signaling and inhibits cell viability of nasopharyngeal carcinoma
}

\author{
YOUHU WANG ${ }^{1}$, WENYAO $\mathrm{JIN}^{2}$ and JUNBO WANG ${ }^{3,4}$ \\ ${ }^{1}$ Department of Otolaryngology-Head and Neck Surgery, The First Hospital of Lanzhou University; \\ ${ }^{2}$ The First Clinical Medical College; ${ }^{3}$ School of Public Health, Lanzhou University, Lanzhou, Gansu 730000; \\ ${ }^{4}$ School of Basic Medical Science, Peking University, Beijing 100191, P.R. China
}

Received November 2, 2020; Accepted February 24, 2021

DOI: $10.3892 / \mathrm{mmr} .2021 .12010$

\begin{abstract}
Nasopharyngeal carcinoma (NPC) is a common disease with high prevalence worldwide, affecting hundreds of thousands of patients every year. Although its progress can be inhibited by concurrent chemoradiotherapy and platinum-based agents, there is also a need for novel drugs to treat NPC. The present study identified tanshinone IIA as a potent drug that could suppress the proliferation of HK1 cells by enhancing pyroptosis via regulation of the miR-125b/foxp3/caspase-1 signaling pathway. Firstly, the effects of tanshinone IIA on HK1 cells were assessed and it was confirmed that treatment with tanshinone IIA significantly decreased the proliferation of HK1 cells, with increased activity of caspase- 3 and caspase-9. Then, the pyroptosis levels after tanshinone IIA administration were detected. The results showed that tanshinone IIA enhanced pyroptosis in a dose-dependent manner. Furthermore, the mechanism underlying the effects of tanshinone IIA on HK1 cells were explored. It was found that transfection with a microRNA (miR)-125b agomir and a small interfering RNA (si)-foxp3 plasmid reversed the inhibitory effect induced by tanshinone IIA, accompanied by an increase in reactive oxygen species levels and lactate dehydrogenase release, indicating a critical role of $\mathrm{miR}-125 \mathrm{~b} / \mathrm{foxp} 3$ signaling in pyroptosis in HK1 cells. In conclusion, the present study demonstrates that tanshinone IIA enhances pyroptosis and inhibits the proliferation of HK1 cells by modulating $\mathrm{miR}-125 \mathrm{~b} /$ foxp3/caspase-1/GSDMD signaling. It is the first study to reveal the inhibitory effect of tanshinone IIA on HK1 cells and to demonstrate the critical role of miR-125b/foxp3 signaling in mediating these effects, providing robust evidence for the treatment of NPC.
\end{abstract}

Correspondence to: Professor Youhu Wang, Department of Otolaryngology-Head and Neck Surgery, The First Hospital of Lanzhou University, 1 Donggangxi Road, Lanzhou, Gansu 730000, P.R. China

E-mail: 623809834@qq.com

Key words: tanshinone IIA, nasopharyngeal carcinoma, pyroptosis, microRNA-125, foxp3

\section{Introduction}

Nasopharyngeal carcinoma (NPC) is an epithelial carcinoma arising from the nasopharynx epithelium (1) and is characterized by a high prevalence, especially in south China, southeastern Asia and Africa (2), affecting 130,000 patients worldwide in 2018 (3). A study identified environmental factors, Epstein-Barr virus infection and genetic susceptibility as risk factors for NPC (4). At present, the application of radiotherapy and optimization of chemotherapy strategies have greatly contributed to improvements in survival rates in patients with NPC (3). However, these treatments have some limitations, including complications and potential adverse effects (5). Therefore, there is an urgent need to explore novel drugs to treat NPC in clinic settings.

Tanshinone IIA is a major component of Salvia miltiorrhiz and exhibits various physiological activities, including anti-tumor, anti-inflammatory and antioxidant effects. In a hypoxic microenvironment, tanshinone IIA was shown to decrease hypoxia-inducible factor $\alpha$ expression and suppress the secretion of vascular endothelial growth factor and basic fibroblast growth factor by regulating the b-catenin/TCF3/LEF1 signaling pathway, leading to the inhibition of proliferation, tube formation and metastasis of human umbilical vein endothelial cells (6). In addition, tanshinone IIA inhibited PI3K/Akt/FoxO1 signaling and alleviated blast-induced inflammation and oxidative stress, leading to improvement in lung blast injuries (7). Importantly, treatment with tanshinone IIA significantly suppressed proliferation and induced apoptosis in various cancer cell types, including ovarian cancer (8), cervical cancer (9) and gastric cancer (10). For instance, Tong et al (11) demonstrated that tanshinone IIA could promote the expression of microRNA (miR)-145 and enhance pyroptosis of HeLa cells, resulting in decreased proliferation of these cells. Notably, tanshinone IIA could also downregulate the expression of miR30b and initiate apoptosis in HepG2 cells (12). These findings reveal a critical role of miRs in mediating the effects of tanshinone IIA; this prompted further exploration of their association.

Recent research has focused on programmed cell death in the treatment of tumors. A lytic and inflammatory type of regulated cell death, pyroptosis requires membrane-damaging gasdermin proteins and is characterized by swelling and 
lysis of cells and release of several pro-inflammatory factors, including interleukin (IL)-18, IL-1 $\beta$ and caspase-3 (13). Caspase- 3 is one of the decisive markers of apoptosis, and is positively associated with some inflammatory factors, such as CD68, tumor necrosis factor $\alpha$ and IL-1b. Additionally, Gasdermin D (GSDMD) is the pyroptotic substrate of caspase- 1 and belongs to the conserved gasdermin family. The gasdermin-N domains in GSDMD bind to membrane lipids and perforate the membrane, leading to the disruption of osmotic potential and further causing cell swelling, with large bubbles blowing from the plasma membrane (13). It has been demonstrated that p53 directly enhances lipopolysaccharide (LPS)-induced pyroptosis to markedly decrease tumor growth in A549 cells, suggesting a critical role of p53 in inhibiting tumor growth in patients (14). Notably, a series of studies have focused on the anticancer effects of natural molecules, including tanshinone IIA (11), omega-3 docosahexaenoic acid (15) and paclitaxel (16). However, how tanshinone IIA regulates pyroptosis in NPC cells remains elusive.

In the present study, the effects of tanshinone IIA on HK1 cells were detected and their apoptotic levels, including the activity of intracellular caspase-3 and caspase-9, was assessed. The expression of GSDMD and caspase-1 was also evaluated. Furthermore, the role of $\mathrm{miR}-125 \mathrm{~b} /$ foxp 3 signaling in the activity of tanshinone IIA was explored by transfecting cells with a miR-125b agomir and small interfering RNA (si)-foxp3 plasmid.

\section{Materials and methods}

Cell culture and chemicals. Human NPC cell line HK1 was obtained from The Cell Bank of Type Culture Collection of The Chinese Academy of Sciences. HK1 cells were seeded in DMEM (cat. no. C11995500BT; Gibco; Thermo Fisher Scientific, Inc.) containing 10\% fetal bovine serum (cat. no. P30-3301; PAN-Biotech) and $1 \%$ penicillin-streptomycin (cat. no. 15140-122; Gibco; Thermo Fisher Scientific, Inc.) in a water-saturated atmosphere at $37^{\circ} \mathrm{C}$. Tanshinone IIA (Fig. 1A) was procured from Selleck Chemicals (cat. no. S2365). Dimethyl sulfoxide (cat. no. 276855; Sigma-Aldrich; Merck KGaA) was used as a negative control.

Cell transfection. The miR-125b agomir, si-foxp3 plasmid (constructed in pEGFP-N2), and their negative controls (pEGFP-N2) were obtained from Guangzhou RiboBio Co., Ltd. The plasmid was resuspended to a final concentration of $1 \mu \mathrm{g} / \mu \mathrm{l}$. HK1 cells $\left(1 \times 10^{6}\right.$ cells $\left./ \mathrm{ml}\right)$ were cultured in six-well plates for $24 \mathrm{~h}$. According to the manufacturer's instructions, the plasmid and agomir were transfected using Lipofectamine ${ }^{\circledR}$ 2000 reagent (cat. no. 11668-027; Invitrogen; Thermo Fisher Scientific, Inc.). After transfection for $72 \mathrm{~h}$, cells were harvested for further research.

MTT assay. HK1 cells $\left(1 \times 10^{6}\right.$ cells/well) were seeded and cultured in six-well plates for $12 \mathrm{~h}$. Then, the cells were treated with different concentrations of tanshinone IIA for $0,24,48$ and $72 \mathrm{~h}$. MTT assays were performed using a commercial kit according to the manufacturer's instructions (cat. no. M1020; Beijing Solarbio Science \& Technology Co.,
Ltd.). An enzyme-linked immunosorbent assay (ELISA) plate reader was used to determinate the optical density at $490 \mathrm{~nm}$.

Caspase-3 and caspase-9 activity assay. HK1 cells $\left(1 \times 10^{6}\right.$ cells/well) were seeded in six-well plates. Then, total protein was extracted from cells using a cell lysis buffer (cat. no. R0020; Beijing Solarbio Science \& Technology Co., Ltd.). The concentration of protein was detected with a BCA protein assay kit (cat. no. P1511-1, Applygen Technologies, Inc.). According to the manufacturer's instructions, the activity of caspase-3 and caspase-9 was assessed with a Caspase 3 Activity lit (cat. no. BC3830; Beijing Solarbio Science \& Technology Co., Ltd.) and Caspase9 Activity kit (cat. no. BC3890, Beijing Solarbio Science \& Technology Co., Ltd.). A microplate reader was used to determine the absorbance values at $405 \mathrm{~nm}$. The activity levels were expressed relative to the control group.

Western blot analysis. This analysis was performed as described in a previous study (16). Total protein of HK1 cells was extracted using RIPA lysis buffer (cat. no. R0020, Beijing Solarbio Science \& Technology Co., Ltd.) containing $1 \%$ protease inhibitor cocktail and phosphatase inhibitor. The protein concentration of the lysate was detected with a BCA Assay kit (cat. no. P1511-1, Applygen Technologies, Inc.). Protein lysates were resolved by $10 \%$ sodium dodecyl sulfate polyacrylamide gel electrophoresis and then transferred to polyvinylidene fluoride membranes (cat. no. IPVH00010; EMD Millipore). The membranes were blocked with 5\% skimmed milk for $2 \mathrm{~h}$, incubated with primary antibodies, and then with horseradish peroxidase-conjugated secondary antibodies. Proteins were visualized with an ECL western blotting substrate (cat. no. 170-5060; Bio-Rad Laboratories, Inc.) using a Bio-Rad System (Bio-Rad Laboratories, Inc.). Actin served as a loading control. The primary antibodies were diluted at 1:2,000 and the secondary antibodies were diluted at 1:5,000. Antibodies against GSDMD (cat nos. ab210070 and ab215203) and foxp3 (cat. no. ab215206) were obtained from Abcam. The caspase-1 (cat. no. 3866T) antibody was obtained from Cell Signaling Technology, Inc. The cleaved caspase-1 (cat. no. A0964) and actin (cat. no. AC026) antibodies were purchased from ABclonal Biotech Co., Ltd. The secondary antibodies were obtained from Biodragon.

Reverse transcription-quantitative PCR (RT-qPCR). This assay was performed according to the manufacturer's instructions. Briefly, total RNA was isolated from control and treated cells using TRIzol reagent (cat. no. 15596-026; Invitrogen; Thermo Fisher Scientific, Inc.). Then, a cDNA synthesis kit (cat. no. 04896866001; Roche) and SYBR green (TransGen Biotech Co., Ltd.) were used to determine the relative expression of target genes with the ABI-Quant Studio 5 system (Thermo Fisher Scientific, Inc.). The mRNA expression levels of the target genes were normalized to $\beta$-actin expression. The relative mRNA expression was calculated using $2^{-\Delta \Delta C q}(17,18)$. The primer pairs used in the present study are listed in Table SI.

ELISA assays. HK1 cells ( $1 \times 10^{5}$ cells/well) were cultured in 24-well plates and treated with tanshinone IIA, miR-125b agomir, or si-foxp3 plasmid. Levels of IL-1b and IL-18 were 

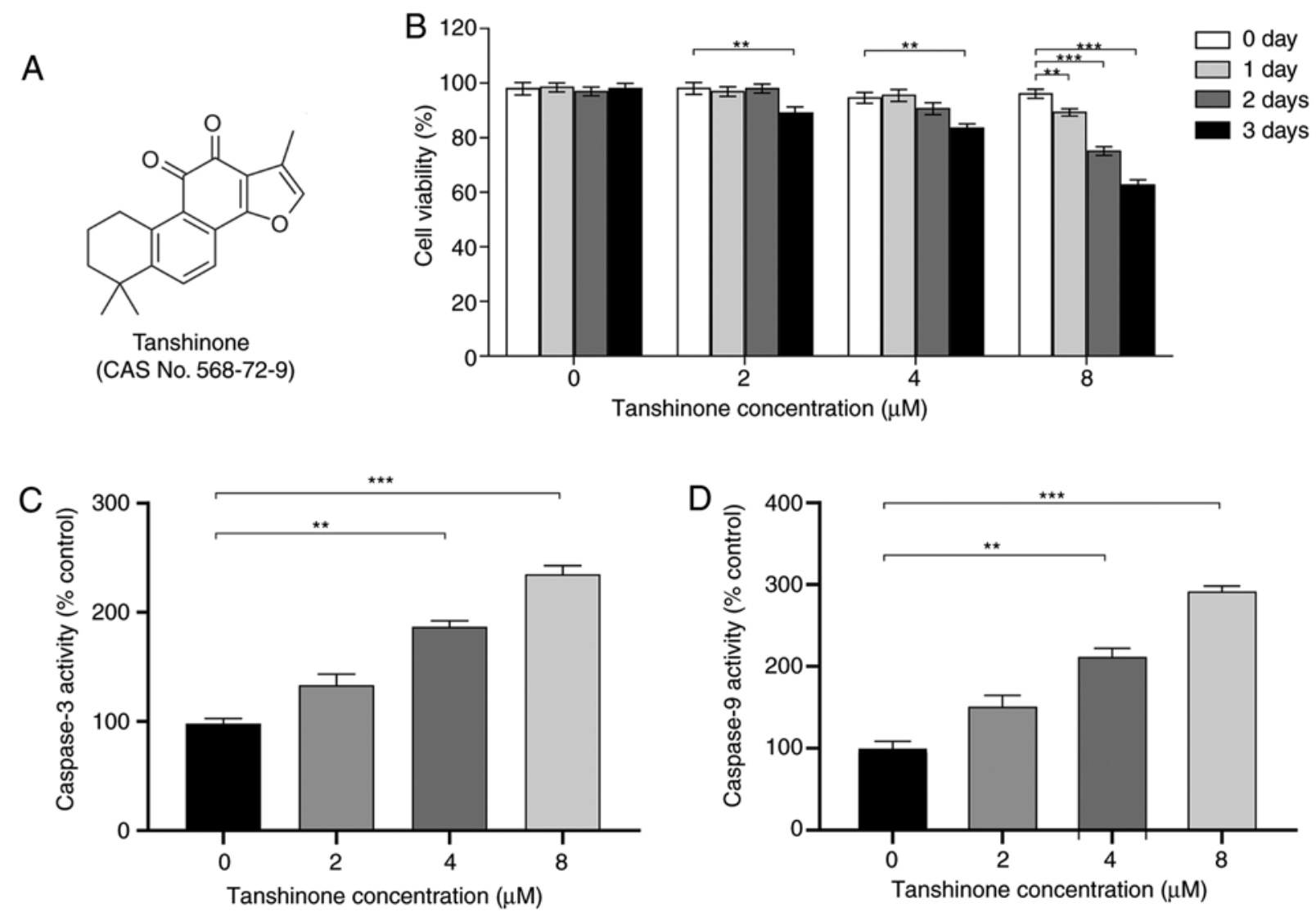

Figure 1. Tanshinone IIA inhibits the proliferation of HK1 cells by enhancing apoptosis. (A) Structure of tanshinone IIA. (B) Viability of HK1 cells after tanshinone IIA treatment. (C and D) Relative activity of caspase-3 and caspase- 9 in HK1 cells. ${ }^{* *} \mathrm{P}<0.01 ;{ }^{* * *} \mathrm{P}<0.005$. All experiments were repeated at least three times.

measured using commercially available ELISA kits following the manufacturer's instructions (cat. nos. ab214025 and ab215539; Abcam). Experiments were repeated three times.

Statistical analysis. For statistical analyses, the results were expressed as mean \pm SEM. Statistical significance was determined by two-tailed student's t-test or one-way analysis of variance followed by Bonferroni analysis (for data meeting homogeneity of variance) or Tamhane's T2 analysis (for data demonstrating heteroscedasticity). $\mathrm{P}<0.05$ was considered to indicate a statistically significant difference. Statistical calculations were performed using the SPSS software (version 24.0; IBM Corp.).

\section{Results}

Tanshinone IIA inhibits cell proliferation and enhances apoptosis of HK1 cells. Previous studies have demonstrated the anticancer effects of tanshinone IIA in cervical cancer $(9,11)$. In the present study, the effects of tanshinone IIA on HK1 cells were assessed (Fig. 1A). The results showed that the proliferation of HK1 cells was significantly inhibited following tanshinone IIA treatment, in a dose- and time-dependent manner. Compared with controls, HK1 cells treated with tanshinone IIA for $72 \mathrm{~h}$ showed markedly decreased viability. In addition, the inhibitory effect on HK1 cells was gradually enhanced with increasing concentration of tanshinone IIA (Fig. 1B). Moreover, the apoptotic levels of HK1 cells treated with tanshinone IIA were detected. Caspase-3 and caspase-9 activity was notably increased compared with that of control cells after treatment for $72 \mathrm{~h}$ (Fig. 1C and D), suggesting enhanced apoptosis of HK1 cells. These results demonstrate that tanshinone IIA upregulated the apoptotic levels of NPC cells, leading to a decrease in cell proliferation.

Tanshinone IIA enhances pyroptotic level of HKI cells. According to a previous study, HK1 was treated with $8 \mu \mathrm{M}$ tanshinone IIA for $72 \mathrm{~h}$ (11). Considering the potential effects of tanshinone IIA on pyroptosis, it was assessed whether administration of tanshinone IIA regulated pyroptosis in HK1 cells. As expected, the expression of caspase-1 and GSDMD was significantly upregulated by treatment with tanshinone IIA, according to the results of the RT-qPCR assay (Fig. 2A). Protein levels of cleaved GSDMD and caspase-1 were also increased (Fig. 2B and C), indicating enhanced pyroptosis of HK1 cells after tanshinone IIA treatment. Moreover, intracellular ROS levels and LDH release were assessed. Compared with control cells, administration of tanshinone IIA elevated ROS levels and promoted LDH release (Fig. 2D and E). Pyroptosis enhances the secretion of inflammatory cytokines such as IL-18 and IL-1 $\beta$. Therefore, the levels of cleaved IL-18 and IL-1 $\beta$ were further detected in HK1 cells treated with tanshinone IIA by ELISA. The results showed that the expression levels of IL-18 and IL-1 $\beta$ were markedly increased (Fig. 2F), consistent with the results of a previous study (11). Notably, tanshinone IIA exhibits the opposite effect 

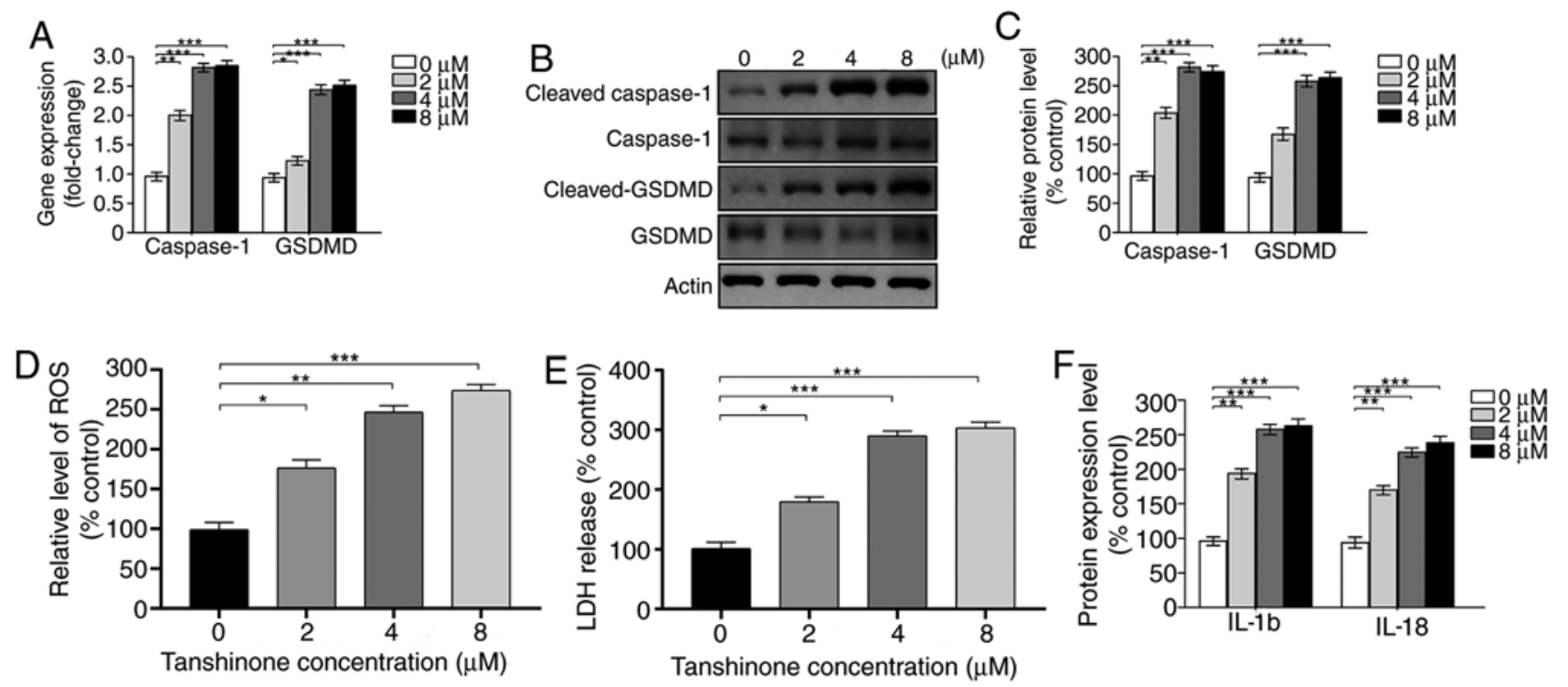

Figure 2. Tanshinone IIA enhances pyroptosis of HK1 cells. (A) Reverse transcription-quantitative PCR analyses of the relative mRNA levels of caspase-1 and GSDMD in HK1 cells. Gene expression was normalized to the $\beta$-actin mRNA level. (B and C) Representative western blot analysis and quantification of cleaved caspase-1 and GSDMD in HK1 cells. Protein expression was normalized to $\beta$-actin levels. (D) Relative ROS levels in HK1 cells after tanshinone IIA treatment. (E) Relative levels of LDH release in HK1 cells after tanshinone IIA treatment. (F) Enzyme-linked immunosorbent assay of IL-1 $\beta$ and IL-18 in HK1 cells after tanshinone IIA treatment. " $\mathrm{P}<0.05 ;{ }^{* *} \mathrm{P}<0.01 ;{ }^{* * * *} \mathrm{P}<0.001$. All experiments were repeated at least three times. GSDMD, gasdermin $\mathrm{D}$; ROS, reactive oxygen species; LDH, lactate dehydrogenase; IL, interleukin.

on normal cells and tumor cells, which may attribute to the different microenvironment. For example, tanshinone IIA attenuates atherosclerosis by inhibiting NLR family pyrin domain containing 3 (NLRP3) inflammasome activation in macrophages (19), while the expression of IL- $1 \beta$ and IL18 was inhibited in Hela cells after tanshinone II A treatment (11). Briefly, tanshinone IIA enhanced pyroptosis in HK1 cells.

Tanshinone IIA enhances pyroptosis by regulating miR-125b. In a previous study, Batool et al (17) demonstrated that miR-125b acts as a tumor suppressor by regulating intrinsic properties of testicular germ cell tumors. In the present study, the association between miR-125b and the anticancer effects of tanshinone IIA were explored. It was found that tanshinone IIA decreased miR-125b levels in a dose-dependent manner. Compared with cells without treatment, administration of tanshinone IIA suppressed the expression level of miR-125b (Fig. 3A). Subsequently, miR-125b agomir was transfected into HK1 cells to explore the role of miR-125b in the activity of tanshinone IIA. The expression of miR-125b was significantly increased after agomir transfection (Fig. 3B). The miR-125b agomir reversed the inhibitory effect of tanshinone IIA on the proliferation of HK1 cells (Fig. 3C). Furthermore, the effect of miR-125b on pyroptosis was assessed after tanshinone IIA treatment. It was found that compared with the control groups, the expression levels of cleaved GSDMD and caspase-1 were significantly decreased after transfection with the miR-125b agomir, according to the results of RT-qPCR and western blotting (Fig. 3D and E). Moreover, the results showed that overexpression of miR-125b decreased the increase in reactive oxygen species (ROS) levels and LDH release (Fig. 3F and G). Given that pyroptosis induces the secretion of IL-18 and IL-1 $\beta$, the levels of cleaved IL- $1 \beta$ and IL-18 in HK1 cells were detected after transfection with the miR-125b agomir. As expected, overexpressing mir-125b inhibited the increase in protein levels of IL-1 $\beta$ and IL-18 induced by tanshinone IIA treatment (Fig. 3H). These results indicate that tanshinone IIA promotes pyroptosis in HK1 cells by regulating miR-125b.

Tanshinone IIA facilitates pyroptosis by regulating the miR-125b/foxp3 signaling pathway. It is well known that miR-125b negatively regulates foxp3 and promotes autophagy in thyroid cancer, thereby enhancing the efficacy of cisplatin (20). In the present study, the expression of foxp3 in HK1 cells was detected after tanshinone IIA treatment. Compared with controls, HK1 cells treated with $8 \mu \mathrm{M}$ tanshinone IIA exhibited elevated foxp3 expression (Fig. 4A and B). Then, HK1 cells were transfected with the si-foxp3 plasmid. The expression of foxp3 was remarkably downregulated after plasmid transfection (Fig. 4C). The results showed that the anti-foxp3 plasmid was able to reverse the inhibition of proliferation of HK1 cells after tanshinone IIA treatment (Fig. 4D). Similarly, plasmid transfection induced a decrease in cleaved GSDMD and caspase-1 expression compared with cells treated with tanshinone IIA (Fig. 4E and F). Moreover, transfection with the si-foxp3 plasmid led to decrease in intracellular ROS levels and LDH release (Fig. 4G and $\mathrm{H}$ ). Notably, the expression of cleaved IL-18 and IL-1 $\beta$ was downregulated after treatment of tanshinone IIA and si-foxp3 plasmid transfection (Fig. 4I). Collectively, these results indicate that tanshinone IIA enhances pyroptosis by regulating miR-125b/foxp3 signaling and inhibits the proliferation of $\mathrm{HK} 1$ cells.

\section{Discussion}

NPC is characterized by a specific geographic distribution, affecting $>130,000$ patients worldwide (3). At present, a combination of concurrent chemoradiotherapy 
A
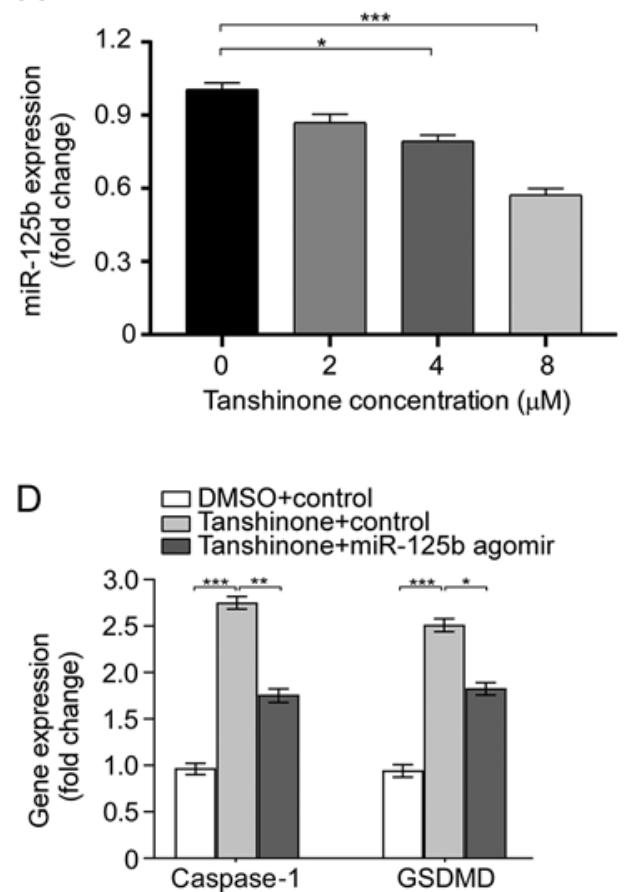

G

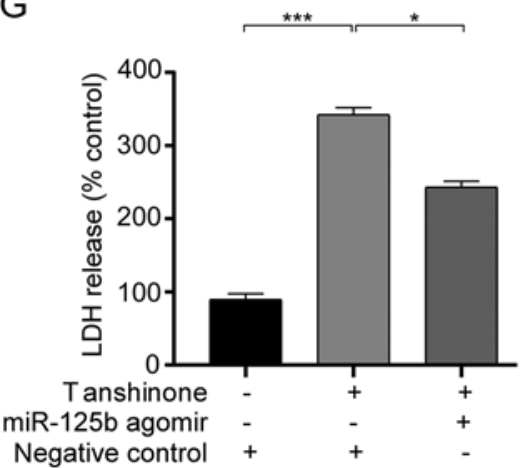

B

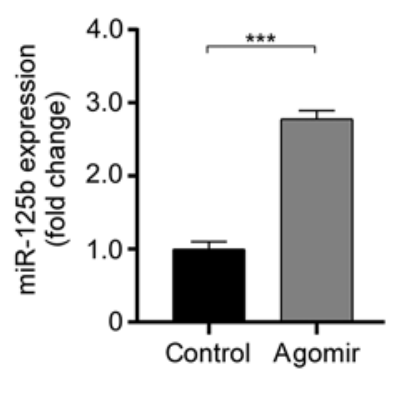

E

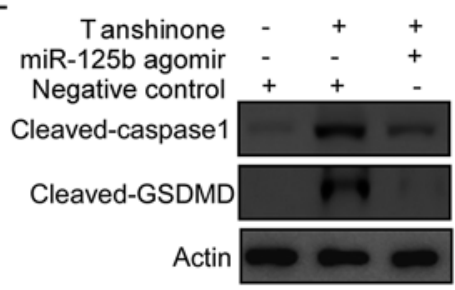

H DMSO+control Tanshinone+control $\square$ Tanshinone+miR-125b agomir

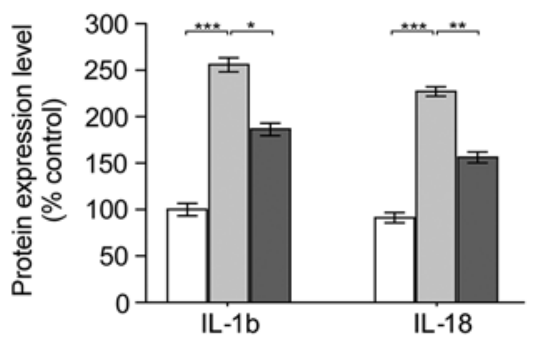

C

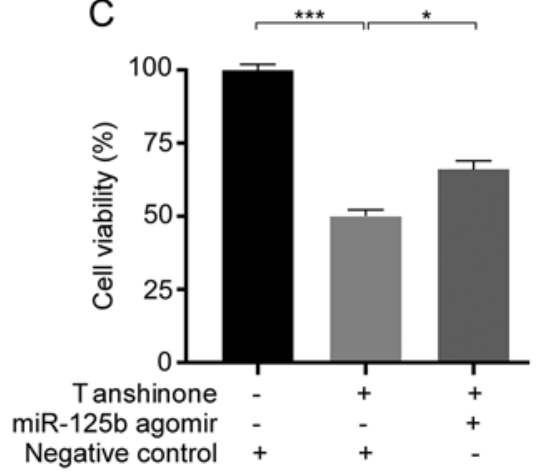

F

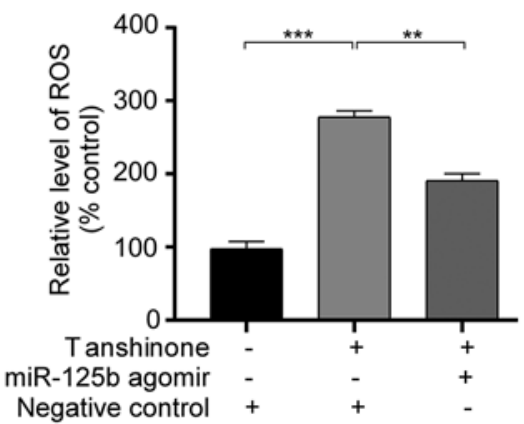

Figure 3. Role of miR-125b in the effects of tanshinone IIA on HK1 cells. (A) Expression of miR-125b in HK1 cells after tanshinone IIA treatment. (B) Expression of miR-125b after transfection with miR-125b agomir. (C) Proliferation of HK1 cells after transfection with miR-125b agomir. (D) Reverse transcription-quantitative PCR analyses of relative mRNA levels of GSDMD and caspase-1 in HK1 cells after transfection with miR-125b agomir. Gene expression was normalized to $\beta$-actin mRNA level. (E) Representative western blot analysis of cleaved GSDMD and caspase-1 in HK1 cells after transfection with miR-125b agomir. Protein expression was normalized to $\beta$-actin levels. (F) Analysis of ROS levels in HK1 cells after transfection with miR-125b agomir. (G) Analysis of LDH release in HK1 cells after transfection with miR-125b agomir. (H) Enzyme-linked immunosorbent assay of IL-1 $\beta$ and IL-18 in HK1 cells after transfection with miR-125b agomir. ${ }^{*} \mathrm{P}<0.05 ;{ }^{* *} \mathrm{P}<0.01 ;{ }^{* * *} \mathrm{P}<0.001$. All experiments were repeated at least three times. GSDMD, gasdermin $\mathrm{D}$; miR, microRNA; ROS, reactive oxygen species; LDH, lactate dehydrogenase; IL, interleukin.

and platinum-based agents is the main clinical approach used to treat NPC (21); however, this leads to side effects including rhinosinusitis (22), dysphagia (23) and transient granulocytopenia (24). Therefore, researchers aim to identify novel natural molecules to inhibit cell proliferation and tumor growth in NPC. In the present study, tanshinone IIA was identified as a potential drug that suppresses cell proliferation of HK1 cells and explored its underlying mechanism. The results showed that the modulation of miR-125b/foxp3/caspase-1 signaling by tanshinone IIA enhances pyroptotic levels in HK1 cells. Tanshinone IIA was also shown to enhance the inflammatory level in HK1 cells, which is opposite to the effect on normal cells. This phenomenon may attribute to the different microenvironment in cells. For example, tanshinone IIA attenuates atherosclerosis by inhibiting NLRP3 inflammasome activation in macrophages (19), while the expression of IL-1 $\beta$ and IL18 was inhibited in HeLa cells after tanshinone II A treatment (11). Briefly, the present study provides evidence to support tanshinone IIA as a potent drug to treat NPC.

Foxp3 is a transcription factor belonging to the forkhead-winged-helix family and is centrally involved in the establishment and maintenance of the Treg cell phenotype (25). In general, foxp $3^{+}$Treg cells can secrete a series of anti-inflammatory cytokines, express co-inhibitory molecules, and modulate the activity of antigen-presenting cells (26). Various studies have clarified a critical role of foxp3 in the treatment of tumors including non-small-cell lung cancer (NSCLC) (27), thyroid cancer (20) and breast cancer (28). For instance, foxp3 overexpression significantly 
A

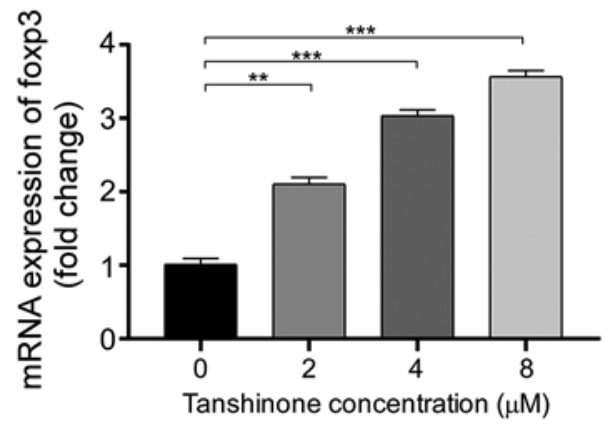

D
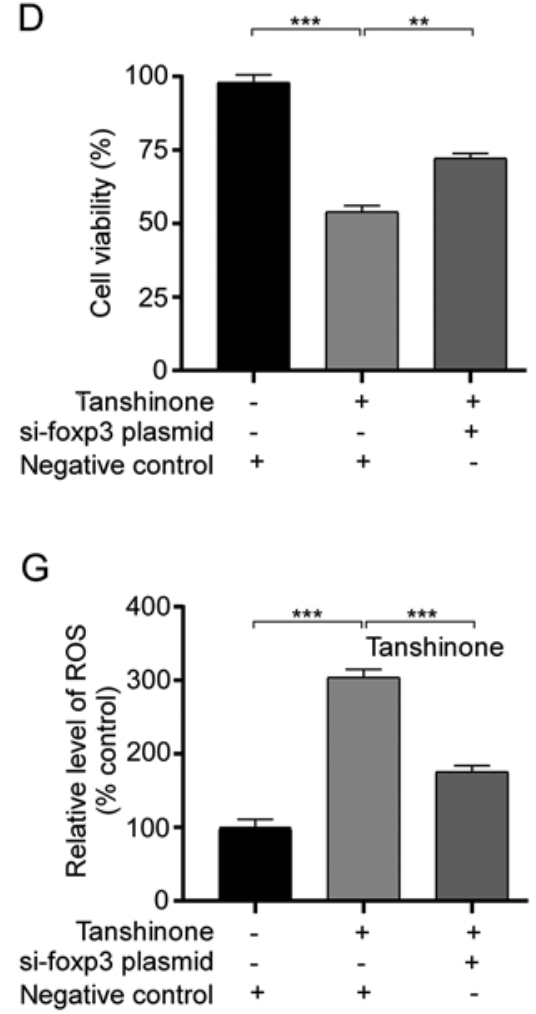

G Negative control
B
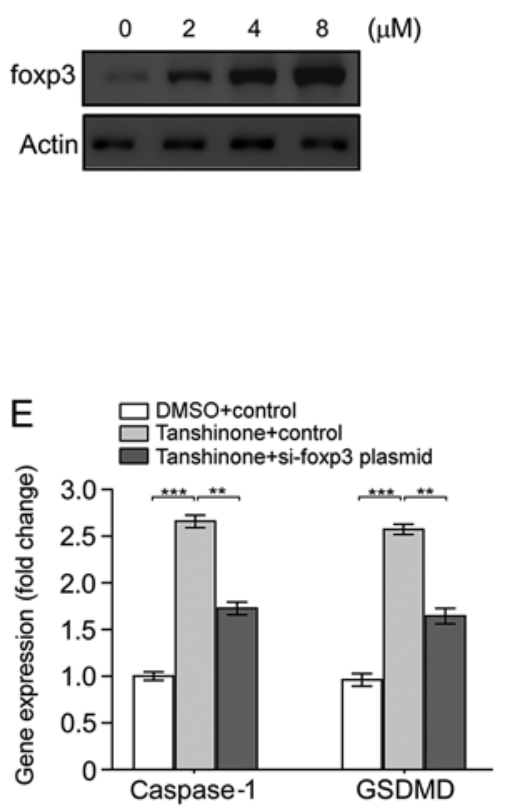

(1)

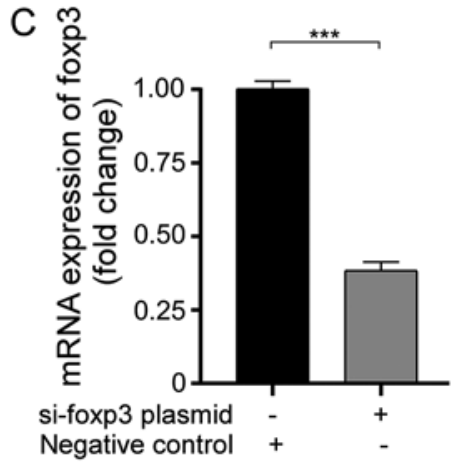

$\mathrm{F}$

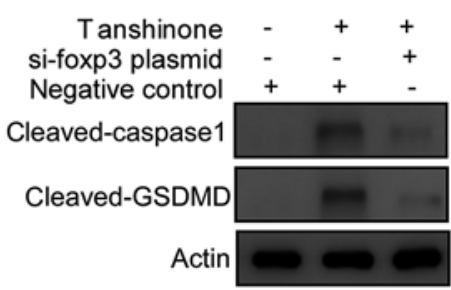

Figure 4. Role of foxp3 in the effects of tanshinone IIA on HK1 cells. (A) RT-qPCR analysis of foxp3 in HK1 cells after tanshinone IIA treatment. (B) Representative western blot analysis of foxp3 in HK1 cells after tanshinone IIA treatment. Protein expression was normalized to $\beta$-actin levels. (C) Expression level of foxp3 after si-foxp3 plasmid transfection. (D) Proliferation of HK1 cells after transfection with si-foxp3 plasmid. (E) RT-qPCR analyses of relative mRNA levels of GSDMD and caspase-1 in HK1 cells after transfection with si-foxp3 plasmid. Gene expression was normalized to $\beta$-actin mRNA levels. (F) Representative western blot analysis of cleaved GSDMD and caspase-1 in HK1 cells after transfection with si-foxp3 plasmid. Protein expression was normalized to $\beta$-actin levels. (G) Analysis of ROS levels in HK1 cells after transfection with si-foxp3 plasmid. (H) Analysis of LDH release in HK1 cells after transfection with si-foxp3 plasmid. (I) Enzyme-linked immunosorbent assay of IL-1 $\beta$ and IL-18 in HK1 cells after transfection with si-foxp3 plasmid. " $\mathrm{P}<0.05 ;{ }^{* *} \mathrm{P}<0.01 ;{ }^{* * *} \mathrm{P}<0.001$. All experiments were repeated at least three times. GSDMD, gasdermin D; si, small interfering RNA; RT-qPCR, reverse transcription-quantitative PCR; ROS, reactive oxygen species; IL, interleukin; LDH, lactate dehydrogenase.

induced cell proliferation, migration and invasion by regulating the Wnt/b-catenin signaling pathway in NSCLC cells, which led to the identification of foxp3 as a co-activator that could facilitate tumor growth and metastasis in NSCLC. However, a study showed that foxp3 acted as a critical suppressor of breast cancer by interacting with Gal-1 (29). Therefore, the precise role of foxp3 in the progress of tumor is still elusive. Notably, studies have shown a positive association of foxp3 with IL-1 $\beta$ (30) and IL-18 (31), which prompted us to explore whether foxp3 was involved in the regulation of pyroptosis. In the present study, it was found that tanshinone IIA upregulated foxp3 expression in HK1 cells. Furthermore, si-foxp3 was transfected into HK1 cells to precisely assess the role of foxp3 on the effect of tanshinone IIA. As expected, plasmid transfection of si-foxp3 reversed the inhibitory effects of tanshinone IIA on HK1 cells, including an increase in cell proliferation, a decrease in intracellular ROS levels, and a decrease in LDH release. Notably, si-foxp3 inhibited pyroptosis of HK1 cells following treatment with tanshinone IIA, indicating an important role of foxp3 in mediating the protective effects of tanshinone IIA. In future, the role of foxp3 in the progress of NPC will be further explored from the aspect of epigenetics, in order to identify other novel molecules to treat NPC in the clinic.

miRs are a class of non-coding RNA that have been shown to have key roles in cell physiology, including in cell differentiation, proliferation and survival (32). miRs 
can bind to complementary target mRNAs and induce inhibition or degradation of the mRNA (33). It has been well demonstrated that miRs are potential targets in the treatment of cancer. In several types of cancer, the expression of certain miRs is markedly associated with poor patient outcomes (34). For example, Jin et al (35) reported that $\mathrm{miR} 15 \mathrm{a} / 16$ targeted and inhibited the components of the transforming growth factor- $\beta$ signaling pathway in the LNCaP cell line; this finding provided potential drug targets for the treatment of prostate cancer. In addition, Hong et al (36) found abnormally high levels of miR-663b in colorectal cancer, and a miR-663b inhibitor exerted an inhibitory effect on the proliferation of colorectal cancer cells by regulating the TNK1/Ras/Raf signaling pathway. In addition to the finding that foxp3 overexpression suppresses the proliferation of tumor cells, it has been shown that miR-125b can directly interact with foxp3 by binding to its 3 '-untranlsated region and inhibit its expression, leading to increased autophagy and enhanced efficacy of cisplatin in thyroid cancer (20). Moreover, researchers have identified a series of natural molecules that can depress tumor growth by downregulating the expression of miR-125b, including ganoderma lucidum polysaccharides (37), camptothecin (38) and silibinin (39). Thus, the role of miR-125b on the effect of tanshinone IIA in HK1 cells was explored. Firstly, it was found that tanshinone IIA downregulated the expression of miR-125b in HK1 cells. Furthermore, transfection with the miR-125b agomir reversed the increase in pyroptosis of HK1 cells induced by tanshinone IIA, with increased expression of GSDMD and caspase-1, elevation of ROS levels and LDH release, and upregulation of IL-18 and IL-1 $\beta$, suggesting that miR-125b/foxp3 signaling was closely involved in mediating the effect of tanshinone IIA. In addition, the role of the miR-125b/foxp3 signaling pathway in apoptosis was investigated after tanshinone IIA administration. The results showed that transfection with the miR-125b agomir or si-foxp3 plasmid reversed the effects of tanshinone IIA on HK1 cells (data not shown), demonstrating miR-125b/foxp3 signaling as a potential novel drug target for the treatment of NPC.

In conclusion, tanshinone IIA was identified as a novel molecule that could inhibit cell proliferation of HK1 cells by regulating miR-125b/foxp3/caspase-1/GSDMD signaling. Tanshinone IIA treatment elevated the pyroptotic levels in HK1 cells. However, transfection withs miR-125b agomir and si-foxp3 plasmids reversed the protective effects of tanshinone IIA. To our knowledge, this is the first study to assess the effects of tanshinone IIA on the expression of miR-125b and to explore the critical role of miR-125b/foxp3 signaling in the regulation of pyroptosis in HK1 cells. In future, the effects of tanshinone IIA on NPC will be explored in vivo, providing a potential approach to treat NPC in the clinic.

\section{Acknowledgements}

Not applicable.

\section{Funding}

No funding was received.

\section{Availability of data and materials}

The datasets generated and analyzed during the present study are available from the corresponding author on reasonable request.

\section{Authors' contributions}

YW designed and performed the study, analyzed the data, and wrote the manuscript. WJ was involved in conducting the experiments, analyzing the data, and writing the manuscript. JW contributed to the writing of the manuscript and data analysis. All authors read and approved the final manuscript.

\section{Ethics approval and consent to participate}

Not applicable.

\section{Patient consent for publication}

Not applicable.

\section{Competing interests}

The authors declare that they have no competing interests.

\section{References}

1. Chua MLK, Wee JTS, Hui EP and Chan ATC: Nasopharyngeal carcinoma. Lancet 387: 1012-1024, 2016.

2. Bray F, Ferlay J, Soerjomataram I, Siegel RL, Torre LA and Jemal A: Global cancer statistics 2018: GLOBOCAN estimates of incidence and mortality worldwide for 36 cancers in 185 countries. CA Cancer J Clin 68: 394-424, 2018.

3. Zhang Y, Chen L, Hu GQ, Zhang N, Zhu XD, Yang KY, Jin F, Shi M, Chen YP, Hu WH, et al: Gemcitabine and cisplatin induction chemotherapy in nasopharyngeal carcinoma. $\mathrm{N}$ Engl J Med 381: 1124-1135, 2019.

4. Chen YP, Chan ATC, Le QT, Blanchard P, Sun Y and Ma J: Nasopharyngeal carcinoma. Lancet 394: 64-80, 2019.

5. Tang LQ, Chen DP, Guo L, Mo HY, Huang Y, Guo SS, Qi B, Tang QN, Wang P, Li XY, et al: Concurrent chemoradiotherapy with nedaplatin versus cisplatin in stage II-IVB nasopharyngeal carcinoma: An open-label, non-inferiority, randomised phase 3 trial. Lancet Oncol 19: 461-473, 2018.

6. Sui H, Zhao J, Zhou L, Wen H, Deng W, Li C, Ji Q, Liu X, Feng Y, Chai $\mathrm{N}$, et al: Tanshinone IIA inhibits $\beta$-catenin/VEGF-mediated angiogenesis by targeting TGF- $\beta 1$ in normoxic and HIF- $1 \alpha$ in hypoxic microenvironments in human colorectal cancer. Cancer Lett 403: 86-97, 2017.

7. Liu Y, Tong C, Tang Y, Cong P, Liu Y, Shi X, Shi L, Zhao Y, Jin H, Li J and Hou M: Tanshinone IIA alleviates blast-induced inflammation, oxidative stress and apoptosis in mice partly by inhibiting the PI3K/Akt/FoxO1 signaling pathway. Free Radic Biol Med 152: 52-60, 2020.

8. Li N, Yang L, Zhang B and Chen S: Tanshinone IIA effects on ovarian cancer cell line. J Pharm Pharmacol 70: 1369-1377, 2018.

9. Liu Z, Zhu W, Kong X, Chen X, Sun X, Zhang W and Zhang R: Tanshinone IIA inhibits glucose metabolism leading to apoptosis in cervical cancer. Oncol Rep 42: 1893-1903, 2019.

10. Xu Z, Chen L, Xiao Z, Zhu Y, Jiang H, Jin Y, Gu C, Wu Y, Wang L, Zhang W, et al: Potentiation of the anticancer effect of doxorubicinin drug-resistant gastric cancer cells by tanshinone IIA. Phytomedicine 51: 58-67, 2018.

11. Tong W, Guo J and Yang C: Tanshinone II A enhances pyroptosis and represses cell proliferation of HeLa cells by regulating miR-145/GSDMD signaling pathway. Biosci Rep 40: BSR20200259, 2020. 
12. Ren X, Wang C, Xie B, Hu L, Chai H, Ding L, Tang L, Xia Y and Dou X: Tanshinone IIA induced cell death via miR30b-p53-PTPN11/SHP2 signaling pathway in human hepatocellular carcinoma cells. Eur J Pharmacol 796: 233-241, 2017.

13. Wang Y, Gao W, Shi X, Ding J, Liu W, He H, Wang K and Shao F: Chemotherapy drugs induce pyroptosis through caspase-3 cleavage of a gasdermin. Nature 547: 99-103, 2017.

14. Zhang T, Li Y, Zhu R, Song P, Wei Y, Liang T and Xu G: Transcription factor $\mathrm{p} 53$ suppresses tumor growth by prompting pyroptosis in non-small-cell lung cancer. Oxid Med Cell Longev 2019: 8746895, 2019.

15. Pizato N, Luzete BC, Kiffer LFMV, Corrêa LH, de Oliveira Santos I, Assumpcão JAF, Ito MK and Magalhães KG: Omega-3 docosahexaenoic acid induces pyroptosis cell death in triple-negative breast cancer cells. Sci Rep 8: 1952, 2018.

16. Zeng QZ, Yang F, Li CG, Xu LH, He XH, Mai FY, Zeng CY, Zhang CC, Zha QB and Ouyang DY: Paclitaxel enhances the innate immunity by promoting NLRP3 inflammasome activation in macrophages. Front Immunol 10: 72, 2019

17. Batool A, Wang YQ, Hao XX, Chen SR and Liu YX: A miR-125b/CSF1-CX3CL1/tumor-associated macrophage recruitment axis controls testicular germ cell tumor growth. Cell Death Dis 9: 962,2018

18. Livak KJ and Schmittgen TD: Analysis of relative gene expression data using real-time quantitative PCR and the 2(-Delta Delta C(T)) method. Methods 25: 402-408, 2001.

19. Wen J, Chang Y, Huo S, Li W, Huang H, Gao Y, Lin H, Zhang J, Zhang Y, Zuo Y, et al: Tanshinone IIA attenuates atherosclerosis via inhibiting NLRP3 inflammasome activation. Aging (Albany NY) 13: 910-932, 2020

20. Wang S, Wu J, Ren J, Vlantis AC, Li MY, Liu SYW, Ng EKW, Chan ABW, Luo DC, Liu Z, et al: MicroRNA-125b interacts with Foxp3 to induce autophagy in thyroid cancer. Mol Ther 26 : 2295-2303, 2018

21. Blanchard P, Lee A, Marguet S, Leclercq J, Ng WT, Ma J, Chan AT, Huang PY, Benhamou E, Zhu G, et al: Chemotherapy and radiotherapy in nasopharyngeal carcinoma: An update of the MAC-NPC meta-analysis. Lancet Oncol 16: 645-655, 2015.

22. Kamel R, Al-Badawy S, Khairy A, Kandil T and Sabry A: Nasal and paranasal sinus changes after radiotherapy for nasopharyngeal carcinoma. Acta Otolaryngol 124: 532-535, 2004

23. Jiang $L$, Huang $C$, Gan $Y$, Wu T, Tang $X$, Wang $Y$, Wang $R$ and Zhang Y: Radiation-induced late dysphagia after intensitymodulated radiotherapy in nasopharyngeal carcinoma patients: A dose-volume effect analysis. Sci Rep 8: 16396, 2018.

24. Tsujii H, Kamada T, Tsuji H, Takamura A, Matsuoka Y, Usubuchi $\mathrm{H}$ and Irie G: Improved results in the treatment of nasopharyngeal carcinoma using combined radiotherapy and chemotherapy. Cancer 63: 1668-1672, 1989

25. Lu L, Barbi J and Pan F: The regulation of immune tolerance by FOXP3. Nat Rev Immunol 17: 703-717, 2017.

26. Vignali DA, Collison LW and Workman CJ: How regulatory T cells work. Nat Rev Immunol 8: 523-532, 2008.

27. Yang S, Liu Y, Li MY, Ng CSH, Yang SL, Wang S, Zou C, Dong Y, Du J, Long X, et al: FOXP3 promotes tumor growth and metastasis by activating $\mathrm{Wnt} / \beta$-catenin signaling pathway and EMT in non-small cell lung cancer. Mol Cancer 16: 124, 2017.
28. Zhang G, Zhang W, Li B, Stringer-Reasor E, Chu C, Sun L, Bae S, Chen D, Wei S, Jiao K, et al: MicroRNA-200c and microRNA-141 are regulated by a FOXP3-KAT2B axis and associated with tumor metastasis in breast cancer. Breast Cancer Res 19: 73, 2017.

29. Gao Y, Li X, Shu Z, Zhang K, Xue X, Li W, Hao Q, Wang Z, Zhang W, Wang $S$, et al: Nuclear galectin-1-FOXP3 interaction dampens the tumor-suppressive properties of FOXP3 in breast cancer. Cell Death Dis 9: 416, 2018.

30. Germanidis G, Argentou N, Hytiroglou P, Vassiliadis T, Patsiaoura K, Germenis AE and Speletas M: Liver FOXP3 and PD1/PDL1 expression is down-regulated in chronic HBV hepatitis on maintained remission related to the degree of inflammation. Front Immunol 4: 207, 2013.

31. Yang C, Huang XR, Fung E, Liu HF and Lan HY: The regulatory T-cell transcription factor Foxp3 protects against crescentic glomerulonephritis. Sci Rep 7: 1481, 2017.

32. Tang L, Chen Y, Xiang Q, Xiang J, Tang Y and Li J: The association between IL18, FOXP3 and IL13 genes polymorphisms and risk of allergic rhinitis: A meta-analysis. Inflamm Res 69: 911-923, 2020.

33. Rupaimoole R and Slack FJ: MicroRNA therapeutics: Towards a new era for the management of cancer and other diseases. Nat Rev Drug Discov 16: 203-222, 2017.

34. Bartel DP: MicroRNAs: Genomics, biogenesis, mechanism, and function. Cell 116: 281-297, 2004.

35. Jin W, Chen F, Wang K, Song Y, Fei X and Wu B: miR-15a/miR-16 cluster inhibits invasion of prostate cancer cells by suppressing TGF- $\beta$ signaling pathway. Biomed Pharmacother 104: 637-644, 2018.

36. Hong S, Yan Z, Wang H, Ding L, Song Y and Bi M: miR-663b promotes colorectal cancer progression by activating Ras/Raf signaling through downregulation of TNK1. Hum Cell 33: 104-115, 2020.

37. Li A, Shuai X, Jia Z, Li H, Liang X, Su D and Guo W: Ganoderma lucidum polysaccharide extract inhibits hepatocellular carcinoma growth by downregulating regulatory $\mathrm{T}$ cells accumulation and function by inducing microRNA-125b. J Transl Med 13: 100, 2015.

38. Zeng CW, Zhang XJ, Lin KY, Ye H, Feng SY, Zhang $\mathrm{H}$ and Chen YQ: Camptothecin induces apoptosis in cancer cells via microRNA-125b-mediated mitochondrial pathways. Mol Pharmacol 81: 578-586, 2012.

39. Hossainzadeh S, Ranji N, Naderi Sohi A and Najafi F: Silibinin encapsulation in polymersome: A promising anticancer nanoparticle for inducing apoptosis and decreasing the expression level of $\mathrm{miR}-125 \mathrm{~b} / \mathrm{miR}-182$ in human breast cancer cells. J Cell Physiol 234: 22285-22298, 2019.

This work is licensed under a Creative Commons Attribution-NonCommercial-NoDerivatives 4.0 International (CC BY-NC-ND 4.0) License. 